Article

\title{
Can Implicit Measures Augment Suicide Detection in Youth? The Feasibility and Acceptability of the Death Implicit Association Test among Pediatric Medical Inpatients
}

\author{
Annabelle M. Mournet ${ }^{1}$, Daniel S. Powell ${ }^{1}$, Elizabeth C. Lanzillo ${ }^{1}$, Sandra McBee-Strayer ${ }^{2}$, Emory Bergdoll ${ }^{2}$, \\ Catherine R. Glenn ${ }^{3}$, Alexander Millner ${ }^{4}$, Maryland Pao ${ }^{1}$, Matthew K. Nock ${ }^{4}$, Lisa M. Horowitz ${ }^{1, *(D)}$ \\ and Jeffrey A. Bridge ${ }^{2,5}$
}

1 Office of the Clinical Director, National Institute of Mental Health, Bethesda, MD 20892, USA; amm883@psych.rutgers.edu (A.M.M.); danpowell2@gmail.com (D.S.P.); lanzillo@cua.edu (E.C.L.); paom@mail.nih.gov (M.P.)

2 The Abigail Wexner Research Institute at Nationwide Children's Hospital, Columbus, OH 43215, USA; sandra.mcbee-strayer@nationwidechildrens.org (S.M.-S.); ebergdoll16@gmail.com (E.B.); jeff.bridge@nationwidechildrens.org (J.A.B.)

3 Department of Psychology, Old Dominion University, Norfolk, VA 23529, USA; cglenn@odu.edu

4 Department of Psychology, Harvard University, Cambridge, MA 02138, USA; amillner@fas.harvard.edu (A.M.); nock@wjh.harvard.edu (M.K.N.)

5 College of Medicine, The Ohio State University, Columbus, OH 43210, USA

* Correspondence: horowitzl@mail.nih.gov

Citation: Mournet, A.M.; Powell, D.S.; Lanzillo, E.C.; McBee-Strayer, S.; Bergdoll, E.; Glenn, C.R.; Millner, A.; Pao, M.; Nock, M.K.; Horowitz, L.M.; et al. Can Implicit Measures Augment Suicide Detection in Youth? The Feasibility and Acceptability of the Death Implicit Association Test among Pediatric Medical Inpatients. Adolescents 2022, 2, 44-52. https:// doi.org/10.3390/adolescents2010006

Academic Editor: Laura L. Hayman

Received: 3 January 2022

Accepted: 7 February 2022

Published: 9 February 2022

Publisher's Note: MDPI stays neutral with regard to jurisdictional claims in published maps and institutional affiliations.

Copyright: (c) 2022 by the authors. Licensee MDPI, Basel, Switzerland. This article is an open access article distributed under the terms and conditions of the Creative Commons Attribution (CC BY) license (https:/ / creativecommons.org/licenses/by/ $4.0 /)$.

\begin{abstract}
Background: Medically ill youth are at increased suicide risk, necessitating early detection. This study aimed to assess the feasibility of administering the Death Implicit Association Test (Death IAT) to pediatric medical inpatients. Methods: Participants completed measures including the Ask Suicide-Screening Questions (ASQ) and the Death IAT. Results: Over 90\% of participants found the Death IAT to be acceptable and more than $75 \%$ of participants were comfortable completing the task. There was a small, but statistically significant, improvement from pre-survey to post-survey reports of $\operatorname{mood}(t(174)=3.02, p=0.003, d=0.15)$. Participants who endorsed a past suicide attempt on the ASQ had significantly higher "suicide" trial D-scores than those without a past suicide attempt (Wilcoxon $W=1312 ; p=0.048 ; d=0.61)$. Conclusions: Implementing an IAT measure among pediatric medical inpatients was feasible and acceptable. In exploratory analyses, "suicide" trial IAT D-scores were associated with past suicide attempts, suggesting future studies should examine whether implicit measures may be useful in hospital settings to augment detection of youth suicide risk.
\end{abstract}

Keywords: suicide risk; mental health screening; Death Implicit Association Test; youth; pediatric medical inpatient

\section{Introduction}

Suicide is a growing public health crisis and the second leading cause of death among youth [1]. Screening for suicide risk among pediatric medical inpatients allows youth at risk for suicide to be identified early and connected to mental health resources. While many adolescents will report suicidal ideation when asked directly through self-report screening, some may be reluctant to discuss thoughts or plans [2]. Studies of suicide decedents have shown that approximately $80-90 \%$ of youth saw a healthcare provider in the year prior to their death by suicide [3].

Risk factors for youth suicidal thoughts and behaviors include history of suicidal thoughts and behaviors [4], depressive symptoms, and diagnosis of mental health disorders [5], as well as medical illnesses [6]. Despite the increased suicide risk for medically hospitalized patients with physical illnesses [6], few screening tools exist for patients outside of psychiatric units and those that do exist rely on self-report. Given the transient 
nature of suicidal thoughts and behaviors (STB) [7], screening could be further improved by incorporating complimentary measures that do not rely on self-report.

One test that is not reliant on self-report measurement is the Implicit Association Test (IAT), which assesses individuals' implicit attitudes and subconscious associations (unconscious) that may be sensitive or stigmatized and, therefore, might not be reliably self-reported [8]. Several versions of the IAT have been developed, including the Death IAT [9], which measures implicit self-identification with death (including suicide).

Numerous studies have demonstrated associations between stronger self-identification with death (as measured by the Death IAT) and more frequent and severe STB among adults [9-12]. Few studies, however, have looked at the utility of the Death IAT among youth and all have been limited to psychiatric samples [13-16]. Dickstein and colleagues [13] found that adolescent participants with non-suicidal self-injury had stronger identification with death versus life than participants with suicide attempt histories. Similarly, Miller et al. [16] identified that adolescent suicide attempters in psychiatric inpatient units had stronger implicit identification with death, compared with non-suicidal controls. Studies by Glenn et al. $[14,15]$ observed that, for adolescents admitted to psychiatric units, stronger implicit identification with death at psychiatric admission was associated with recent suicidal ideation, but not with suicidal behavior. Death IAT scores were also higher among adolescents with prior attempts who reattempted suicide over a year-long follow-up period [15].

No previous studies have examined the utility and feasibility of the Death IAT among pediatric medical inpatients. Extant research has shown generally positive patient, parent, and clinician support for use of explicit mental health screening tools in pediatric medical settings [17]. However, there is notably less research regarding patient opinions on implicit measures. While recent research by Cha and colleagues [18] has refuted concerns about the iatrogenic risk of completing suicide-related IATs, a slight decline in participant mood following completion of the IAT was detected. It is unclear whether responding to a computer task like the Death IAT could create discomfort in medically hospitalized pediatric patients, especially those with potentially life-threatening diseases who may experience anxiety related to their illness and an actual possibility of death [19]. To explore clinical utilization of the Death IAT, especially among a medically ill population who may experience heightened discomfort with talking about death, due to their medical illness, it is important to understand if this type of IAT causes emotional distress or is well tolerated. If the Death IAT can be administered to pediatric medical inpatients without causing distress, it has the potential to augment suicide risk screening in a way that does not rely on self-report bias and can provide valuable clinical information regarding patient suicide risk.

The aim of this study was to determine the feasibility and acceptability of utilizing the Death IAT among pediatric medical inpatients. For this study, feasibility was assessed by pediatric patient opinions regarding the acceptability of the Death IAT and whether completion of the Death IAT alters a patient's mood. An additional exploratory aim was to assess whether implicit self-identification with death/suicide is associated with the outcome (positive or negative) of a self-reported suicide risk screen and whether individuals with prior suicide attempts have increased implicit self-identification with death/suicide compared to those without a suicide attempt history.

\section{Materials and Methods}

\subsection{Participants and Procedures}

Participants were pediatric inpatients (ages 10-21 years) admitted to a medical or surgical inpatient unit at an urban children's hospital as part of a larger suicide risk screening instrument validation study [20]. Common reasons for admittance included general medical conditions, such as fever and stomach pain, as well as general surgery and injuries. A convenience sample of youth were recruited between January 2017 and January 2019. The age cut off of 21 years was determined based on the American Academy of Pediatrics (AAP) guidelines on the classification of adolescents [21]. Exclusion criteria 
included being non-English speaking, imminent discharge from the unit (within the same day), no guardian available to provide consent (if the patient was under the age of 18), and cognitive impairment (determined based on nursing report, parent report, and/or ability to answer questions). Participants were administered a battery of self-report questionnaires, which included a demographic questionnaire and the Ask Suicide-Screening Questions (ASQ) [22], followed by the Death IAT and a pre/post survey for the Death IAT. The Death IAT was always administered following the ASQ in order to preserve the integrity of the larger instrument validation study's aim to validate the ASQ among pediatric medical patients. Participants who screened positive for suicide risk were given a follow-up mental health assessment and patient safety actions were taken as clinically indicated. This study was approved by the institutional review board (IRB) of the data collection site and of the site coordinating the larger validation study. For participants aged 17 years or younger, informed assent and parental permission were obtained for all youth. Informed consent was obtained directly from participants aged 18 years or older. Additional information regarding study recruitment is reported by Horowitz, Wharff, et al. [20].

\subsection{Measures}

The ASQ consists of four yes/no items and was developed to rapidly detect suicide risk among pediatric medical patients [22]. Endorsement of any item or refusal to answer any question prompts a fifth question: "Are you having thoughts of killing yourself right now?" Responding "no" to the first four items of the ASQ results in a negative screen. A "yes" or refusal to answer any one of the first four items and a "no" response to the fifth item indicates a non-acute positive screen, while a "yes" to this item indicates imminent risk and is considered an acute positive screen. The ASQ was developed among pediatric patients in the emergency department, using the Suicidal Ideation Questionnaire [23] as the gold standard. The ASQ has since been validated among pediatric patients in inpatient medical/surgical units [20], outpatient specialty and primary care clinics [24], as well as adult medical patients [25]. Among pediatric medical inpatients, the ASQ has strong psychometric properties among pediatric medical inpatients, with a sensitivity of $96.7 \%$, and a specificity of $91.1 \%$ [20].

The Death IAT [9] is a 5-min computerized task to assess implicit self-identification with death and suicide. Using a standard keyboard, participants categorize semantic stimuli to the left or right based on the categories at the top of the screen ("ME" vs. "NOT ME"; and "LIFE" vs. "DEATH"). The same semantic stimuli originally reported in Nock et al. [9] were utilized. The task contains two blocks, each consisting of 40 trials and additional practice trials. Block A pairs "ME" with "LIFE" ("NOT ME" and "DEATH") on the same side of the screen and Block B pairs "ME" with "DEATH". For each participant, a standardized $\mathrm{D}$-score is derived using the difference in reaction time between Block A and Block B. A faster response on the "ME" / DEATH" block (Block B) compared to the "ME" / "LIFE" block (Block A) is assumed to suggest a stronger self-identification with death, such that positive D-scores denote a stronger association between death and self, while negative D-scores are assumed to represent a stronger association between life and self. Participants with potentially invalid data, such as data with too many errors (30\% overall or $40 \%$ in one block) or too many really slow ( $>10 \mathrm{~s})$ or really fast trials $(<400 \mathrm{~ms})(10 \%$ of overall trials or $25 \%$ of trials in one block), were excluded. The Death IAT was administered using Inquisit Lab 4 [26] (Figure 1). 
ME

or

LIFE

\section{NOT ME}

or

DEATH

\section{Suicide}

Figure 1. Death IAT Sample Item.

The Death IAT pre/post-survey was developed for this study to assess participant opinions of the Death IAT. Using a 5-point Likert scale, the Death IAT pre-survey consists of three items that assess the participant's mood prior to completion of the task. Participants rate their current mood, level of sadness, and extent to which they feel worried or anxious. Mood is assessed from 1 (negative) to 5 (positive) while sadness and feeling worried or anxious are scored from 1 (not at all) to 5 (extremely). The first three items of the Death IAT post-survey are identical to the three items of the Death IAT pre-survey. The subsequent three items assess the participant's own comfort with completing the Death IAT and their opinions on the acceptability of administering the Death IAT to youth in the hospital. Face validity of this tool was determined by the study team. The items assessing mood, sadness, worry, and anxiety were developed based on the items used by Cha and colleagues [18] to assess iatrogenic effects of the Death IAT. The items used to assess comfort and acceptability were created based on items used in previous research involving assessment of feasibility in studies using the ASQ $[17,25,27]$.

\subsection{Data Analytic Strategy}

Descriptive statistics from the feasibility questionnaire are reported. Repeated-means t-tests were used to analyze changes in reported mood, worry/anxiety, and sadness from pre-survey to post-survey. Standardized D-scores were computed for each participant. Pearson correlations were computed to assess correlations between self-reported mood and Death IAT scores. To account for non-normally distributed data, Wilcoxon rank sum tests for unpaired data were used to compare groups. We compared those who screened positive on the ASQ versus those who screened negative and participants who reported a suicide attempt versus those who did not report a prior suicide attempt on overall D-scores and D-scores only for trials of the word "suicide." The decision to perform exploratory analyses to look at D-scores for the word "suicide" was based on a recent study by Glenn and colleagues [15] where the "suicide" stimuli were found to have the largest effect size for the difference between groups. This may be particularly relevant for medical patients who have stronger self-associations with death in a way that does not directly translate to suicide risk. 


\section{Results}

\subsection{Participant Demographics}

Of the 221 pediatric medical inpatients approached to participate in the study, 200 (90.5\%) enrolled. Twenty-four participants were excluded from this analysis due to not completing the Death IAT or due to potentially invalid Death IAT data, resulting in a final sample of 176 participants. Two participants did not complete the Death IAT pre/post survey, though their ASQ and Death IAT data were retained in the present analyses. The sample was $61.9 \%$ female $(109 / 176), 72.2 \%$ White $(127 / 176)$, and had a mean age of 15.8 years $(S D=2.6)($ Table 1$)$.

Table 1. Demographics of Study Participants $(n=176)$.

\begin{tabular}{lc}
\hline & $\boldsymbol{n} \mathbf{( \% )}$ \\
\hline Gender & \\
Female & $109(61.9 \%)$ \\
Male & $67(38.1 \%)$ \\
Race or ethnicity & \\
White & $127(72.2 \%)$ \\
African American & $25(14.2 \%)$ \\
Hispanic/Latino & $7(4.0 \%)$ \\
Multiple Races & $15(8.5 \%)$ \\
Unknown & $2(1.1 \%)$ \\
Mean Age (SD) & 15.8 years $(2.6)$ \\
10-11 years & $9(5.1 \%)$ \\
12-17 years & $114(64.8 \%)$ \\
18-21 years & $53(30.1 \%)$ \\
\hline
\end{tabular}

\subsection{Participant Opinions and Mood}

After completing the Death IAT, participants were asked about their opinions and comfort with completing a task such as the Death IAT (Table 2). When asked about their own comfort completing the task, the majority of respondents $(79.9 \% ; 139 / 174)$ reported they felt "very comfortable" or "comfortable". When asked whether it is acceptable to ask kids in the hospital to complete a task that involves thinking about life and death, most participants $(90.8 \% ; 158 / 174)$ responded "Yes".

Table 2. Participant Comfort and Acceptability with the Death IAT $(n=174)$.

\begin{tabular}{|c|c|c|c|}
\hline Item & $\begin{array}{c}\text { Very Comfortable/Comfortable } \\
n(\%)\end{array}$ & $\begin{array}{l}\text { Neither Comfortable Nor } \\
\text { Uncomfortable } n(\%)\end{array}$ & $\begin{array}{l}\text { Uncomfortable/Very } \\
\text { Uncomfortable } n(\%)\end{array}$ \\
\hline \multirow[t]{2}{*}{ Comfort completing the task } & $139(79.9 \%)$ & $30(17.2 \%)$ & $5(2.9 \%)$ \\
\hline & $\begin{array}{c}\text { Yes } \\
n(\%)\end{array}$ & $\begin{array}{l}\text { I Don't Know } \\
n(\%)\end{array}$ & $\begin{array}{l}\text { No } \\
n(\%)\end{array}$ \\
\hline $\begin{array}{c}\text { Acceptability of asking kids } \\
\text { in the hospital to complete } \\
\text { this task }\end{array}$ & $158(90.8 \%)$ & $13(7.5 \%)$ & $3(1.7 \%)$ \\
\hline
\end{tabular}

Participant mood was assessed before and after completing the Death IAT task. There was a small but statistically significant improvement from pre-survey $(M=3.97 ; S D=0.94)$ to post-survey reports of $\operatorname{mood}(M=4.11 ; S D=0.92)(t(174)=3.02, p=0.003, d=0.15)$. There was no significant change in reports of worry/anxiety between pre-survey report $(M=1.88$; $S D=1.05)$ and post-survey report $(M=1.88 ; S D=1.03)(t(174)=0.00, p=1.00, d=0.00)$, nor a significant change for reports of sadness between the pre-survey $(M=1.41 ; S D=0.75)$ and post-survey $(M=1.39 ; S D=0.79)(t(174)=-0.90, p=0.37, d=0.03)$. Death IAT scores were not correlated with self-reported pre- or post-survey mood (pre-survey: $r=-0.02$, $p=0.79$; post-survey: $r=-0.07, p=0.39$ ), worry/anxiety (pre-survey: $r=0.11, p=0.15$; 
post-survey: $r=0.11, p=0.16$ ), nor sadness (pre-survey: $r=-0.02, p=0.79$; post-survey: $r=-0.0008, p=1.00$ ). Death IAT and ASQ Results (Table 3).

Table 3. Wilcoxon Rank Sum Tests to compare D-Scores and ASQ Responses $(n=176)$.

\begin{tabular}{|c|c|c|c|c|}
\hline Test & Wilcoxon W & $p$-Value & Cohen's d & $95 \% \mathrm{CI}$ \\
\hline ASQ Screening Outcome vs. Death IAT D-score & 1660 & 0.050 & 0.54 & $-0.0003-0.3105$ \\
\hline Past Suicide Attempt vs. Death IAT D-score & 1301 & 0.063 & 0.59 & $-0.0089-0.3424$ \\
\hline ASQ Screening Outcome vs. "Suicide" Trial D-score & 1598 & 0.092 & 0.42 & $-0.0491-0.7257$ \\
\hline Past Suicide Attempt vs. "Suicide" Trial D-Score & 1312 & 0.048 & 0.61 & $0.0057-1.0617$ \\
\hline
\end{tabular}

ASQ Screening Outcome categorized as positive or negative.

Of the 176 participants who completed the Death IAT, the mean D-score was -0.66 $(S D=0.32$; range $=-1.34-0.35)$. Sixteen participants $(9.1 \% ; 16 / 176)$ screened positive on the ASQ, with all of these individuals screening "non-acute" positive, by endorsing at least one ASQ item, but not the fifth ASQ item. Twelve participants $(6.8 \% ; 12 / 176)$ reported a past suicide attempt.

There were no group differences on Death IAT scores between participants screening positive and those screening negative on the ASQ $(M=-0.51, S D=0.32$ vs. $M=-0.68$, $S D=0.31$; Wilcoxon $W=1660 ; p=0.050)$. Similarly, Death IAT scores did not differ between those with and without a past suicide attempt $(M=-0.48, S D=0.33$ vs. $M=-0.67$, $S D=0.31$; Wilcoxon $W=1301 ; p=0.063)$. Exploratory analyses revealed no significant difference in "suicide" trial D-scores for participants who screened positive on the ASQ compared to screen-negative participants $(M=-0.47, S D=0.86$ vs. $M=-0.81, S D=0.74$; Wilcoxon $W=1598 ; p=0.092)$. However, participants who endorsed a past suicide attempt had significantly higher "suicide" trial D-scores compared to those who did not report a past suicide attempt $(M=-0.30, S D=0.91$ vs. $M=-0.81, S D=0.74$; Wilcoxon $W=1312$; $p=0.048 ; d=0.61)$.

\section{Conclusions}

Implementing an implicit measure, such as the Death IAT, among pediatric medical inpatients was found to be feasible and acceptable. Participant opinions demonstrated acceptability and comfort with the Death IAT task; over $90 \%$ of participants reported that the administration of such a task is acceptable, and more than three quarters of participants were "comfortable" or "very comfortable" completing the task. The high enrollment rate of $90.5 \%$ indicates that most parents were comfortable with their children completing a task related to suicide risk screening.

There is a dearth of research assessing the impact of an IAT on a subject's mood. Although the Project Implicit website [28] states that the IAT "poses the possibility of causing discomfort", few studies, beyond the work of Cha and colleagues [18], have reported on a participant's mood before and after completing an IAT. The present findings suggest that engaging with a task like the Death IAT does not have a negative or harmful impact on medically ill pediatric patients despite their being confronted with words such as "die" and "suicide." Results indicate that even among medically hospitalized youth, repeatedly viewing stimuli related to death did not cause report of worsened mood or emotional discomfort.

Conversely, there was a small increase in positive mood following the Death IAT. While the surveys were kept brief to not overburden participants, the finding of the slight mood increase may be due to the fact that youth enjoy engagement with computer games, even if they involve words like "suicide" and "deceased", or possibly that they were bored in the hospital and enjoyed having something to occupy their time. Though further research is needed to investigate whether this improvement in mood is clinically significant, preliminary findings suggest that an IAT might be an acceptable way to augment screening for suicide risk among young people who are digital natives. 
Given potential concerns regarding the transient nature of suicidal thoughts and behaviors, as well as the stigmatization of suicide risk that may lead to unreliable selfreport of suicide risk, we also aimed to enhance previous research on the use of an IAT to detect risk for suicide by exploring whether implicit self-identification with death was correlated with suicide risk screens and if they could differentiate youth with a history of suicide attempts. The difference in Death IAT scores for participants who screened positive on the ASQ or reported a past suicide attempt compared to participants who screened negative or did not report a past attempt only approached significance. This may be a result of not all the stimuli in the Death IAT being specific to suicide, but generally related to death ("die" vs. "suicide"). Medical patients who are ill may have stronger self-associations with death in a way that does not directly translate to suicide risk. To detect the difference between self-associations with death due to suicide versus other, potentially medical, reasons, "suicide" trials were used. Participants who endorsed a past suicide attempt had significantly higher "suicide" trial D-scores compared to those who did not report a past suicide attempt, denoting a stronger association between "suicide" and self for individuals with a past suicide attempt. The present findings are consistent with prior research in non-medical settings showing that implicit association with death and/or suicide, as measured by the Death IAT, is able to distinguish between suicide attempters and non-suicide attempters $[9-13,15,16]$. The current exploratory findings suggest that this is also true for pediatric medical patients.

This study has several limitations to note. This study was a small sample of medical inpatients from one study site. The low base rate of suicidal thoughts and behaviors in non-psychiatric samples led to relatively few individuals with these thoughts and behaviors and low statistical power. In addition, comfort and mood were assessed with a de novo survey created for this study. The Death IAT was also administered after the ASQ for all participants in order to ensure integrity of the instrument validation portion of the larger study, which may influence responses on the Death IAT. Additionally, this study was not able to assess the severity of participant's medical illness, meaning that there is a lack of information on how medical illness impacted thoughts of suicide in this study.

Future research should assess the use of the Death IAT to longitudinally predict suicidal risk and behavior among hospitalized pediatric medical patients. Because this study was cross-sectional, Death IAT scores were not analyzed in relation to future suicidal thoughts and behaviors post-discharge from the hospital. Prior studies demonstrate the predictive abilities of the IAT $[9,10,14,15,29]$ and suggest that future studies should investigate the prospective use of the Death IAT among medical patients. Additionally, this study focused on medical patients, due to the important need to understand whether medically ill youth could be asked questions about death and suicide without causing emotional distress. Future studies should continue to test feasibility and validity of the Death IAT in additional populations, including the general population. Lastly, future studies should also seek to build upon the exploratory findings of this study to confirm and expand the findings regarding the ability of the Death IAT to augment suicide risk screening.

Repeatedly viewing stimuli about self-associations with death as part of a computerized task was found to be feasible and acceptable among medically hospitalized youth Exploratory findings also suggested that suicide-specific stimuli on the Death IAT were able to identify pediatric medical patients who endorsed a past suicide attempt on the ASQ. While it is important to ask youth directly about suicidal thoughts and behaviors, implicit measures of suicide risk, such as the Death IAT, may be useful in hospital settings to augment suicide risk screening.

Author Contributions: Conceptualization, L.M.H., J.A.B., and M.P.; methodology, L.M.H., J.A.B., M.K.N., and M.P.; software, A.M., J.A.B.; validation, L.M.H., J.A.B., M.K.N., and M.P.; formal analysis, A.M.M., D.S.P., and A.M.; investigation, S.M.-S., E.B., and J.A.B.; resources, A.M., L.M.H., J.A.B., and M.P.; data curation, A.M.M; writing—original draft preparation, A.M.M.; writing—review and editing, D.S.P., E.C.L., C.R.G., A.M., L.M.H., J.A.B., M.K.N., and M.P.; visualization, L.M.H., J.A.B., and M.P.; supervision, L.M.H., J.A.B., M.K.N., and M.P.; project administration, S.M.-S., and E.B.; 
funding acquisition, L.M.H., J.A.B., and M.P. All authors have read and agreed to the published version of the manuscript.

Funding: This research was supported in part by the Intramural Research Program of the NIMH (Annual Report Number ZIAMH002922).

Institutional Review Board Statement: This study was approved by an institutional review board at the National Institutes of Health and Nationwide Children's Hospital.

Informed Consent Statement: All participants provided informed assent and parental permission/consent was obtained for all participants.

Acknowledgments: The authors would like to thank all the patients, nurses, and mental health and medical teams that helped make the study run smoothly. The authors would also like to acknowledge and thank the people who were instrumental to the success of the study, including Elizabeth Ballard, Debbie Snyder, William Booker, Mary Tipton, Nathan Lowry, Jeanne Radcliffe, Elizabeth Wharff, Abigail Ross, Erina White, Martine Solages, Kendra Heck, and Connor Bauer. The views expressed in this abstract are those of the authors and do not reflect the official policy of the National Institute of Mental Health, National Institutes of Health, or the Department of Health and Human Services. ClinicalTrials.gov Identifier NCT02050867.

Conflicts of Interest: The authors have no conflict of interest to report.

\section{References}

1. Centers for Disease Control and Prevention. Web-based Injury Statistics Query and Reporting System. National Center for Injury Prevention and Control, CDC. 2018. Available online: https://www.cdc.gov/injury/wisqars/index.html (accessed on 28 January 2021).

2. Horesh, N.; Zalsman, G.; Apter, A. Suicidal Behavior and Self-Disclosure in Adolescent Psychiatric Inpatients. J. Nerv. Ment. Dis. 2004, 192, 837-842. [CrossRef] [PubMed]

3. Rhodes, A.E.; Khan, S.; Boyle, M.H.; Tonmyr, L.; Wekerle, C.; Goodman, D.; Bethell, J.; Leslie, B.; Lu, H.; Manion, I. Sex Differences in Suicides among Children and Youth: The Potential Impact of Help-Seeking Behaviour. Can. J. Psychiatry 2013, 58, $274-282$. [CrossRef] [PubMed]

4. Cha, C.B.; Franz, P.J.; Guzmán, E.M.; Glenn, C.R.; Kleiman, E.M.; Nock, M.K. Annual Research Review: Suicide among youth-epidemiology, (potential) etiology, and treatment. J. Child. Psychol. Psychiatry 2017, 59, 460-482. [CrossRef] [PubMed]

5. Cash, S.J.; Bridge, J.A. Epidemiology of youth suicide and suicidal behavior. Curr. Opin. Pediatrics 2009, 21, 613-619. [CrossRef] [PubMed]

6. Rodway, C.; Tham, S.-G.; Ibrahim, S.; Turnbull, P.; Windfuhr, K.; Shaw, J.; Kapur, N.; Appleby, L. Suicide in children and young people in England: A consecutive case series. Lancet Psychiatry 2016, 3, 751-759. [CrossRef]

7. Nock, M.K.; Prinstein, M.J.; Sterba, S.K. Revealing the form and function of self-injurious thoughts and behaviors: A real-time ecological assessment study among adolescents and young adults. J. Abnorm. Psychol. 2009, 118, 816-827. [CrossRef] [PubMed]

8. Greenwald, A.G.; McGhee, D.E.; Schwartz, J.L. Measuring individual differences in implicit cognition: The implicit association test. J. Pers. Soc. Psychol. 1998, 74, 1464-1480. [CrossRef] [PubMed]

9. Nock, M.K.; Park, J.M.; Finn, C.T.; Deliberto, T.L.; Dour, H.J.; Banaji, M.R. Measuring the suicidal mind: Implicit cognition predicts suicidal behavior. Psychol. Sci. 2010, 21, 511-517. [CrossRef] [PubMed]

10. Barnes, S.M.; Bahraini, N.H.; Forster, J.E.; Stearns-Yoder, K.A.; Mph, T.A.H.; Smith, G.; Nagamoto, H.T.; Nock, M.K. Moving beyond self-report: Implicit associations about death/life prospectively predict suicidal behavior among Veterans. Suicide Life-Threat. Behav. 2017, 47, 67-77. [CrossRef] [PubMed]

11. Glenn, J.J.; Werntz, A.J.; Slama, S.J.K.; Steinman, S.A.; Teachman, B.A.; Nock, M.K. Suicide and self-injury-related implicit cognition: A large-scale examination and replication. J. Abnorm. Psychol. 2017, 126, 199-211. [CrossRef] [PubMed]

12. Millner, A.J.; Coppersmith, D.D.L.; Teachman, B.A.; Nock, M.K. The Brief Death Implicit Association Test: Scoring recommendations, reliability, validity, and comparisons with the Death Implicit Association Test. Psychol. Assess. 2018, 30, 1356-1366. [CrossRef] [PubMed]

13. Dickstein, D.P.; Puzia, M.E.; Cushman, G.K.; Weissman, A.B.; Wegbreit, E.; Kim, K.L.; Nock, M.K.; Spirito, A. Self-injurious implicit attitudes among adolescent suicide attempters versus those engaged in nonsuicidal self-injury. J. Child. Psychol. Psychiatry 2015, 56, 1127-1136. [CrossRef]

14. Glenn, C.R.; Kleiman, E.M.; Coppersmith, D.; Santee, A.; Esposito, E.C.; Cha, C.B.; Nock, M.K.; Auerbach, R.P. Implicit identification with death predicts change in suicide ideation during psychiatric treatment in adolescents. J. Child Psychol. Psychiatry 2017, 58, 1319-1329. [CrossRef] [PubMed]

15. Glenn, C.R.; Millner, A.J.; Esposito, E.C.; Porter, A.C.; Nock, M.K. Implicit Identification with Death Predicts Suicidal Thoughts and Behaviors in Adolescents. J. Clin. Child Adolesc. Psychol. 2019, 48, 263-272. [CrossRef] [PubMed] 
16. Millner, A.J.; Augenstein, T.M.; Visser, K.H.; Gallagher, K.; Vergara, G.A.; D'Angelo, E.J.; Nock, M.K. Implicit Cognitions as a Behavioral Marker of Suicide Attempts in Adolescents. Arch. Suicide Res. 2019, 23, 47-63. [CrossRef] [PubMed]

17. Ross, A.M.; White, E.; Powell, D.; Nelson, S.; Horowitz, L.; Wharff, E. To Ask or Not to Ask? Opinions of Pediatric Medical Inpatients about Suicide Risk Screening in the Hospital. J. Pediatr. 2016, 170, 295-300. [CrossRef]

18. Cha, C.B.; Glenn, J.J.; Deming, C.A.; D'Angelo, E.J.; Hooley, J.M.; Teachman, B.A.; Nock, M.K. Examining potential iatrogenic effects of viewing suicide and self-injury stimuli. Psychol. Assess. 2016, 28, 1510-1515. [CrossRef] [PubMed]

19. Noyes, R., Jr.; Carney, C.P.; Langbehn, D.R. Specific phobia of illness: Search for a new subtype. J. Anxiety Disord. 2004, 18, 531-545. [CrossRef]

20. Horowitz, L.M.; Wharff, E.A.; Mournet, A.M.; Ross, A.M.; McBee-Strayer, S.; He, J.-P.; Lanzillo, E.C.; White, E.; Bergdoll, E.; Powell, D.S.; et al. Validation and Feasibility of the ASQ Among Pediatric Medical and Surgical Inpatients. Hosp. Pediatr. 2020, 10, 750-757. [CrossRef] [PubMed]

21. American Academy of Pediatrics. Bright Futures: Guidelines for the Health Supervision of Infants, Children, and Adolescents; American Academy of Pediatrics: Elk Grove Village, IL, USA, 2008.

22. Horowitz, L.M.; Bridge, J.A.; Teach, S.J.; Ballard, E.; Klima, J.; Rosenstein, D.L.; Wharff, E.A.; Ginnis, K.; Cannon, E.; Joshi, P.; et al. Ask Suicide-Screening Questions (ASQ). Arch. Pediatr. Adolesc. Med. 2012, 166, 1170-1176. [CrossRef] [PubMed]

23. Reynolds, W.M. Suicidal Ideation Questionnaire (SIQ); Psychological Assessment Resources: Odessa, FL, USA, 1987.

24. Aguinaldo, L.D.; Sullivant, S.; Lanzillo, E.C.; Ross, A.; He, J.P.; Bradley-Ewing, A.; Bridge, J.A.; Horowitz, L.M.; Wharff, E.A. Validation of the ask suicide-screening questions (ASQ) with youth in outpatient specialty and primary care clinics. Gen. Hosp. Psychiatry 2021, 68, 52-58. [CrossRef] [PubMed]

25. Snyder, D.J.; Ballard, E.; Stanley, I.H.; Ludi, E.; Kohn-Godbout, J.; Pao, M.; Horowitz, L.M. Patient Opinions About Screening for Suicide Risk in the Adult Medical Inpatient Unit. J. Ment. Health Adm. 2017, 44, 364-372. [CrossRef] [PubMed]

26. Inquisit; Version 4.0.6; Millisecond Software: Seattle, WA, USA, 2014.

27. Ballard, E.D.; Stanley, I.H.; Horowitz, L.M.; Pao, M.; Cannon, E.A.; Bridge, J.A. Asking youth questions about suicide risk in the pediatric emergency department: Results from a qualitative analysis of patient opinions. Clin. Pediatric Emerg. Med. 2013, 14, 20-27. [CrossRef] [PubMed]

28. Project Implicit. Ethical Considerations. Harvard University. 2011. Available online: https://implicit.harvard.edu/implicit/ ethics.html (accessed on 28 January 2021).

29. Ellis, T.E.; Rufino, K.A.; Green, K.L. Implicit Measure of Life/Death Orientation Predicts Response of Suicidal Ideation to Treatment in Psychiatric Inpatients. Arch. Suicide Res. 2015, 20, 59-68. [CrossRef] [PubMed] 